\title{
COMPARAISON DE LA VARIABILITÉ GÉNÉTIQUE DE DEUX ESPĖCES DE POISSONS, L'OMBRE COMMUN ET LE GARDON, DANS UN FLEUVE AMÉNAGÉ
}

\author{
YVETTE BOUVET, E. PATTEE ET J.L. MASLIN \\ Laboratoire de Biologie Animale et Ecologie, Université Claude Bernard Lyon-I, \\ 43 bd du 11 novembre 1918, F-69622 VILLEURBANNE Cedex \\ (U.R.A. CNRS 1451, Ecologie des Eaux Douces et des Grands Fleuves)
}

\begin{abstract}
RÉSUMÉ
Les deux espèces habitent le bassin du Haut-Rhône français, en amont de Lyon. Mais leur variabilité génétique est très différente. La variabilité intra-populations de l'ombre ne représente qu'un tiers de la variation totale, mais celle du gardon en représente $86 \%$, avec une plus grande diversité dans les annexes du fleuve (Lac du Bourget, bras mort) que dans le chenal. Ces différences sont discutées dans le cadre de l'étendue et de la structure de l'habitat de chaque espèce, ainsi que de l'impact de la gestion piscicole et des aménagements récents. L'ombre constitue de petites communautés autonomes accrochées au terrain et dont la-diversité optimale se situe vers le milieu du gradient amont-aval du bassin. Les annexes semblent constituer, pour le gardon, des réservoirs de diversité génétique à partir desquels un flux génique important mais sélectionné dévale le fleuve.
\end{abstract}

\section{A COMPARISON OF THE GENETIC VARIABILITY OF TWO FISH SPECIES, GRAYLING THYMALLUS THYMALLUS AND ROACH RUTILUS RUTILUS, IN A LARGE REGULATED RIVER}

\section{SUMMARY}

Both species inhabit the catchment of the French Upper Rhone above the city of Lyon (Southeastern France). They mainly differ by their genetic variability : $67 \%$ of overall grayling variability occurs between populations, whereas $86 \%$ of roach variability is to be found within the populations, with a higher diversity in the lateral parts of the system (lake and side arm). These differences are discussed in relation with habitat area and structure, as well as fish management and river equipment. Grayling is organized as small and localized populations, with greatest diversity in the middle of the upstream-downstream gradient, where the environment is most favourable. Backwaters act as genetic reservoirs of roach diversity, from which important but selective fluxes move into the channel and drift downstream, thus maintaining genetic uniformity between populations. 


\section{INTRODUCTION}

La compréhension de la répartition dans l'espace fluvial des populations de poissons, ainsi que de leur évolution en relation avec l'instabilité temporelle de l'hydrosystème se heurtent à une difficulté constante : la définition de l'unité biologique fonctionnelle qui doit être l'objet de l'étude. En effet, dans une zone biogéographique donnée, le caractère hétérogène des regroupements d'individus d'une même espèce peut être la cause d'une mauvaise compréhension de la dynamique de la population étudiée.

L'analyse de la structure génétique d'une espèce par étude du polymorphisme enzymatique permet, comme nous l'avons montré chez le gardon (BOUVET et al., 1991) et l'ombre (BOUVET et al., 1990), d'identifier des sous-ensembles dont le fonctionnement peut alors être suivi dans l'espace et dans le temps.

Nous nous proposons ici de comparer les résultats obtenus dans l'analyse du polymorphisme enzymatique de l'ombre commun et du gardon. Ces deux espèces colonisent une même portion du réseau fluvial étudié : l'amont du bassin du Rhône, mais leurs exigences écologiques divergentes devraient aboutir à une exploitation différente de l'espace fluvial et donc à des différences de structuration de leurs populations.

\section{MATÉRIEL ET MÉTHODES}

Le matériel biologique étudié, l'ombre commun, Thymallus thymallus (L. 1758) et le gardon, Rutilus rutilus ( $\mathrm{L}$. 1758), représente deux espèces de référence en raison de leurs exigences écologiques opposées :

- la première vit exclusivement dans les eaux fraîches à courant vif ; sa température létale supérieure $(24 \mathrm{~h})$ a été évaluée à $26^{\circ} \mathrm{C}$ et sa concentration létale en oxygène dissous $\left(24 \mathrm{~h}-20^{\circ} \mathrm{C}\right)$ à $3,6 \mathrm{mg} \cdot 1^{-1}$ (KRAIEM et PATTEE, 1980). En été, les premières mortalités apparaissent sur le terrain vers $23^{\circ} \mathrm{C}$ (PERSAT, 1976). Sa distribution est ainsi déterminée par la qualité des eaux et leur température. La population du bassin du Haut-Rhône s'appuie sur quelques sous-groupes pérennes se développant dans quelques secteurs à débit réservé, mais surtout dans les principaux affluents (Ain, Furans, Séran) qui alimentent régulièrement le Rhône, de caractère plus artificiel en raison des aménagements (PERSAT, 1988). Une liste de ces derniers est donnée par PATTEE (1988), avec un survol de leurs conséquences.

- la seconde, le gardon, est adaptée aux eaux lentes et stagnantes à température relativement élevée. L'ensemble du réseau hydrographique du Haut-Rhône ne répond pas à ces caractéristiques, ce qui fait que les populations de gardon y sont peu importantes et reposent essentiellement sur un certain nombre de réservoirs alimentant le fleuve (Léman, lac du Bourget, anciens bras du fleuve) et constituant des sites favorables à la reproduction de cette espèce.

Les individus utilisés pour cette étude ont été récoltés par pêche électrique ou aux filets maillants, dans les stations réparties sur le réseau fluvial (fig. 1) pendant les années 1984 à 1990.

Des extraits de divers tissus ont été préparés à partir de ces individus, de manière à réaliser les migrations électrophorétiques sur gel d'amidon. Les protocoles de préparation des extraits et de réalisation des électrophorégrammes sont ceux préconisés par PASTEUR et al. (1987). Les systèmes enzymatiques analysés sont présentés dans le tableau l et représentent 34 locus pour l'ombre commun et 28 locus pour le gardon. L'ensemble des résultats concernant le polymorphisme enzymatique des deux espèces étudiées ici a été publié par BOUVET et al. (1990 et 1991).

Nous considérerons successivement :

- la variabilité génétique intra-population dans chacun des échantillons, évaluée par les taux de polymorphisme et d'hétérozygotie ainsi qu'ils sont définis par PASTEUR (1985).

- les différences et ressemblances entre populations. D'une part, les coefficients de différenciation génétique de NEI (1973) seront utilisés pour évaluer la contribution à la diversité totale $\mathrm{H}_{\mathrm{T}}$ de ses deux composantes diversité intra-population $\mathrm{H}_{S}$ et diversité inter-populations DST. 


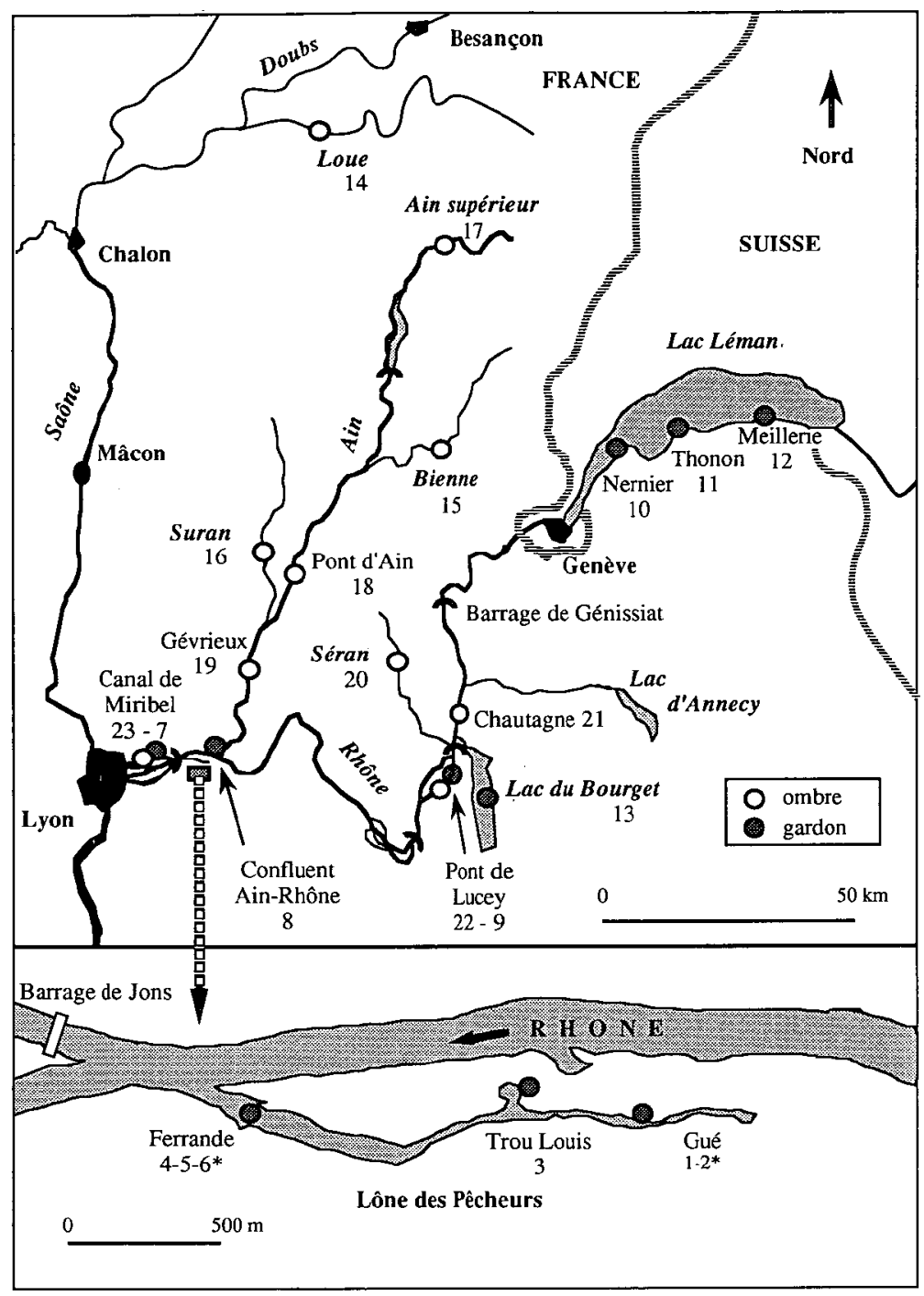

Figure 1 : Echantillonnage des populations de gardon et d'ombre commun.

(*) A la Ferrande et au Gué, chaque numéro représente un prélèvement effectué à une date différente et traité comme une station supplémentaire :

Ferrande 4 : mars 1985

Ferrande 5 : avril 1985

Ferrande 6 : novembre 1984

Gué 1 : avril 1985

Gué 2 : novembre 1984

Figure 1 : Location of the grayling and roach populations investigated.

$\left.{ }^{\star}\right)$ In the side arm (Ferrande and Gué), each number represents a sample collected at a different date and treated as a distinct station :

Ferrande 4 : March 1985

Ferrande 5 : April 1985

Ferrande 6 : November 1984

Gué 1 : April 1985

Gué 2 : November 1984 
Tableau I : Liste des systèmes enzymatiques analysés et des tampons de migration utilisés.

Le nombre d'allèles des locus polymorphes est indiqué entre parenthèses. Tampons de migration (composition : voir PASTEUR et al., 1987) :
MC = Morpholine citrate pH 6,2
TBE 8,6 = Tris borate EDTA pH 8,6
TC $6,7 / 8,0=$ Tris citrate pH 6,7 ou 8,0
TCB = Ridway $=$ Tris citrate borate
TPO4 = Tris phosphate

Table I : Enzymes investigated and buffers used for electrophoretic migrations.

The number of alleles in polymorphoc loci is shown between brackets.

Buffer composition is as in PASTEUR et al. (1987).

\begin{tabular}{|c|c|c|c|c|}
\hline \multirow[t]{2}{*}{$N^{\circ}$ IUBNC } & \multirow[t]{2}{*}{ Enzyme } & \multicolumn{2}{|c|}{ Locus } & \multirow[t]{2}{*}{ Tampons de migration } \\
\hline & & Ombre & Gardon & \\
\hline 2.6.1.1. & AAT & Aat & $\begin{array}{c}\text { Aat.1 } \\
\text { Aat.2 (3) } \\
\end{array}$ & TPO4 \\
\hline 1.1.1.1. & $\mathrm{ADH}$ & Adh & Adh & TCB \\
\hline 2.7.4.3. & AK & Ak & $\begin{array}{l}\text { Ak.1 } \\
\text { Ak.2 }\end{array}$ & MC \\
\hline 2.7.3.2. & CK & $\begin{array}{c}\text { Ck.1 (3) } \\
\text { Ck.2 } \\
\end{array}$ & $\begin{array}{l}\text { Ck.1 } \\
\text { Ck.2 } \\
\end{array}$ & TCB - TPO4 \\
\hline 3.1.1.1. & EST & $\begin{array}{c}\text { Est. 1 (4) } \\
\text { Est.2 }\end{array}$ & $\begin{array}{c}\text { Est.1 (2) } \\
\text { Est.2 (3) } \\
\text { Est.3 }\end{array}$ & TCB \\
\hline 4.2.1.2. & $\mathrm{FH}$ & $\mathrm{Fh}$ & $\mathrm{Fh}$ & $\mathrm{MC}$ \\
\hline 4.4.1.5. & GLO & Glo & --- & TBE 8,6 \\
\hline 1.1.1.8. & GDP & Gdp (2) & Gdp (4) & TPO4 \\
\hline 5.3.1.9. & GPI & $\begin{array}{c}\text { Gpi.1 } \\
\text { Gpi.2 } \\
\text { Gpi.3 } \\
\text { Gpi.4 (2) }\end{array}$ & $\begin{array}{c}\text { Gpi.1 } \\
\text { Gpi.2 (2) }\end{array}$ & TCB \\
\hline 1.1.1.42 & IDHP & $\begin{array}{l}\text { Idhp.1 } \\
\text { Idhp.2 }\end{array}$ & $\begin{array}{c}\text { Idhp.1 } \\
\text { Idhp.2 (3) }\end{array}$ & MC - TPO4 \\
\hline 1.1.1.27 & LDH & $\begin{array}{l}\text { Ldh.1 (2) } \\
\text { Ldh.2 } \\
\text { Ldh.3 (2) } \\
\text { Ldh.4 }\end{array}$ & $\begin{array}{l}\text { Ldh.1. (2) } \\
\text { Ldh.2 } \\
\text { Ldh.3 }\end{array}$ & TCB \\
\hline 1.1 .1 .37 & $\mathrm{MDH}$ & $\begin{array}{l}\text { Mdh.1 } \\
\text { Mdh.2 } \\
\text { Mdh.3 } \\
\end{array}$ & $\begin{array}{l}\text { Mdh.1 (2) } \\
\text { Mdh.2 }\end{array}$ & $\overline{M C}$ \\
\hline 1.1 .1 .40 & MEP & $\begin{array}{l}\text { Mep.1 } \\
\text { Mep.2 }\end{array}$ & $\begin{array}{l}\text { Mep.1 } \\
\text { Mep.2 }\end{array}$ & TPO4 - TC 8,0 \\
\hline 5.3.1.8. & MPI & Mpi & $\begin{array}{c}\text { Mpi.1 } \\
\text { Mpi.2 (2) }\end{array}$ & TC 6,7 - TPO4 \\
\hline 1.1 .1 .43 & PGDH & $\begin{array}{c}\text { Pgdh.1 } \\
\text { Pgdh.2 (2) }\end{array}$ & Pgdh (4) & TC 8,0 - TPO4 \\
\hline 2.7.5.1. & PGM & $\begin{array}{c}\text { Pgm.1 (2) } \\
\text { Pgm.2 (2) } \\
\text { Pgm.3 }\end{array}$ & Pgm (2) & TCB \\
\hline 1.15.1.1. & SOD & $\begin{array}{l}\text { Sod.1 } \\
\text { Sod.2 }\end{array}$ & Sod & TC 6,7 - TCB \\
\hline 1.2.1.37 & $\mathrm{XDH}$ & Xdh & $-\ldots$ & TCB \\
\hline
\end{tabular}




$$
\text { Ainsi : } \quad \mathrm{H}_{\mathrm{T}}=\mathrm{H}_{\mathrm{S}}+\mathrm{D}_{\mathrm{ST}}
$$

D'autre part, nous résumerons les distances génétiques entre populations, basées sur les fréquences alléliques de l'ensemble des locus examinés (NEI, 1972) au moyen de dendrogrammes de classification hiérarchique (UPGMA) (SNEATH et SOKAL, 1973).

Pour préciser davantage la structure des populations de gardon, une analyse factorielle des correspondances multiples a été appliquéeà l'ensemble des génotypes individuels rangés en quatre ensembles regroupant les échantillons issus de milieux hydrologiquement semblables (chenal du Rhône, Lac Léman, Lac du Bourget, Lône des Pêcheurs). La projection des quatre nuages de points sur le plan F1-F2 met en évidence leurs dimensions et leurs positions respectives, illustrant les variabilités respectivement intra- et inter-groupes.

\section{RÉSULTATS}

\section{Taux de polymorphisme et d'hétérozygotie (fig. 2)}

Les valeurs calculées sont plus élevées chez le gardon que chez l'ombre, chez lequel elles sont beaucoup plus hétérogènes.

Le gardon fait preuve d'une remarquable homogénéité, d'où ressortent à peine un polymorphisme peut-être plus faible au confluent de l'Ain et au Canal de Miribel, et une hétérozygotie peut-être plus grande dans le lac du Bourget.
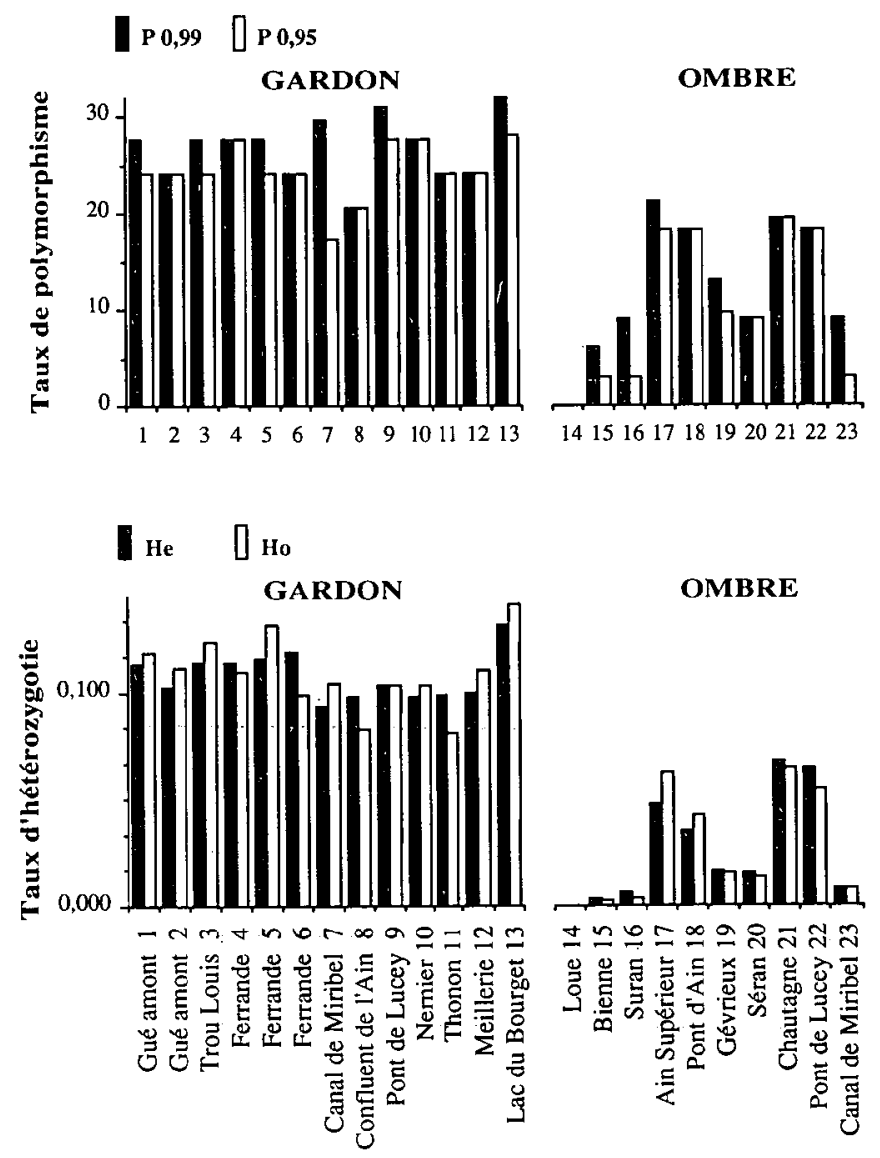

Figure 2 : Polymorphisme et hétérozygotie du gardon et de l'ombre commun. $\mathrm{He}=$ hétérozygotie estimée. $\mathrm{Ho}=$ hétérozygotie observée.

Figure 2 : Roach and grayling polymorphism and heterozygosity. $\mathrm{He}=$ estimated heterozygosity. Ho = observed heterozygosity. 
Il est intéressant d'examiner les données sur l'ombre dans une perspective amont-aval pour chaque sous-système du réseau fluvial. Parmi les stations échantillonnées, la Loue, la Bienne et le Séran représentent les plus petites rivière peuplées par cette espèce en tête de bassin, avec une diversité génétique réduite. Sur les cours d'eau de débit plus important, les valeurs, d'abord élevées, vont en décroissant de l'amont à l'aval, qu'il s'agisse de l'Ain (Ain supérieur - Pont d'Ain - Gévrieux) ou du Rhône (Chautagne - Pont de Lucey - Canal de Miribel). L'échantillon du Suran doit être considéré à part, car il s'agit de la descendance d'un seul couple de géniteurs.

\section{Composantes de la diversité génétique (tableau II)}

L'essentiel de cette diversité provient, chez le gardon, de la variabilité intra-populations $(88,8 \%)$, alors que chez l'ombre elle est due, à parts presque égales, à la variabilité interne aux populations $(57,9 \%)$ et à la variabilité entre populations $(42,1 \%)$.

Tableau II : Composantes de la diversité génétique (NEI, 1973) chez le gardon et l'ombre commun.

$\mathrm{Nb}$ pop = nombre de populations étudiées

$\mathrm{Nb}$ locus $=$ nombre de locus

$\mathrm{H}_{\mathrm{S}} \quad=$ diversité génique moyenne intra-population

DST = diversité génique moyenne entre populations

Table II : Components of genetic diversity according to NEI (1973) in roach and grayling.

$\mathrm{Nb}$ pop = number of populations investigated

$\mathrm{Nb}$ locus = number of loci

$\mathrm{H}_{\mathrm{S}} \quad=$ within-population mean gene diversity

DST = between-populations mean gene diversity

\begin{tabular}{ccccc}
\hline & Nb pop & Nb locus & $\mathrm{H}_{\mathrm{S}} \%$ & $\mathrm{D}_{\mathrm{ST}} \%$ \\
\hline Gardon & 13 & 28 & 88,8 & 11,2 \\
Ombre & 10 & 34 & 57,9 & 42,1 \\
\hline
\end{tabular}

\section{Distances génétiques et classification hiérarchique (fig. 3)}

Chez le gardon, nous observons la forte originalité du lac du Bourget par rapport aux autres stations $(D=0,1)$, et notamment par rapport au Rhône, auquel il n'est relié que par l'étroit canal de Savières. Ces autres stations s'organisent en deux ensemblès relativement proches $(D=0,04)$ regroupant chaque fois des secteurs lénitiques et une partie du chenal: à l'amont, les stations du Léman et le Rhône à Pont de Lucey, à l'aval les stations de la Lône des Pêcheurs et les stations voisines du Rhône, Confluent de l'Ain et Canal de Miribel.

La classification obtenue pour l'ombre est beaucoup moins claire. Aucune association de proximité n'apparaît, comme si la structure spatiale des populations de l'espèce était brouillée : les deux stations du chenal les plus proches (Pont de Lucey-Chautagne) abritent les populations les plus éloignées du point de vue des distances génétiques. La position de deux échantillons provenant du bassin de la Loire (BOUVET et al., 1990) a été représentée pour montrer la grande diversité possible dans l'espèce $T$. thymallus. Sans tenir compte de ce bassin distinct, la diversité inter-populations de l'ombre $(>0,2)$ dépasse encore beaucoup celle du gardon $(\leq 0,1)$. 

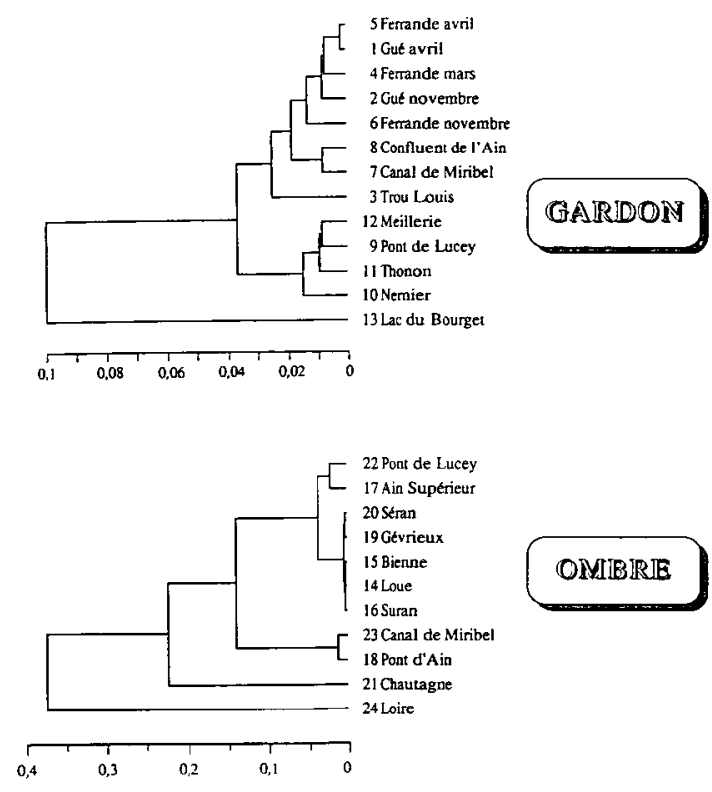

Figure 3 : Distances génétiques entre populations de gardon et d'ombre commun selon NEI (1973).

Figure 3 : Genetic distances between roach and grayling populations, according to NEI (1973).

\section{Analyse multivariée des populations de gardon}

La carte factorielle de la figure 4 explique de façon plus précise la structure de ces populations : celles des deux annexes Lac du Bourget et Lône des Pêcheurs paraissent assez distinctes et montrent une grande diversité relative; seulement une partie de leurs allèles se retrouvent dans l'axe du fleuve (Lac Léman et Rhône), dont les populations sont plus homogènes.

\section{DISCUSSION}

La différence la plus remarquable entre les deux espèces est celle de leurs variabilités génétiques intra- et inter-populations, qui font apparaître l'homogénéité des populations de gardon comparée à l'hétérogénéité des populations d'ombre, alors qu'elles colonisent toutes deux la même zone du réseau fluvial. Ces différences peuvent s'expliquer par les effectifs efficaces (WRIGHT, 1931 ; KIMURA, 1990) plus élevés chez le gardon que chez l'ombre, en raison de leurs caractéristiques démographiques (fécondité, longévité) qui entretiennent des stocks de géniteurs plus importants chez le premier que chez le second. A l'extrême, BOUVET et al. (1990) évaluent à un millier environ les ombres adultes présents dans la Bienne, petit cours d'eau maintenant coupé de l'Ain par un barrage et où polymorphisme et hétérozygotie sont des plus faibles.

Les populations des deux espèces exploitent le réseau fluvial de façon différente, que nous nous sommes efforcés de traduire dans la figure 5 .

Le gardon présente d'abord une faible différenciation amont/aval, ce qui suggère l'existence de flux géniques importants le long du fleuve. La similitude entre les échantillons du lac Léman et de Pont de Lucey, à une centaine de kilomètres de distance, similitude évidente dans les figures 3 (dendrogramme) et 4 (analyse des correspondances), est remarquable. Compte tenu du caractère limnophile et prolifique de l'espèce, le support de ces flux réside sans doute dans la dévalaison d'individus, probablement de nombreux alevins, à travers les pertes du Rhône autrefois, à travers les barrages aujourd'hui. De ce point de vue, le dendrogramme et les taux d'hétérozygotie (figure 2) placent le lac du Bourget à l'écart du fleuve. Mais, par ailleurs, des relations transversales entre le chenal et ses annexes sont suggérées. A l'aval, 
le dendrogramme rapproche bras mort et populations voisines du fleuve. L'analyse des correspondances présente les annexes comme des réservoirs de diversité génétique dont seulement une partie se retrouve dans le fleuve. Ces relations sont étayées par les migrations transversales de géniteurs observées au moment du frai.

Au manque d'autonomie des populations locales de gardon s'oppose l'originalité des populations individuelles d'ombre, fortement soumises, semble-t-il, à la dérive génétique. Les populations de tête de réseau (Loue, Bienne, et, dans une moindre mesure, Séran) et celles de l'aval (Canal de Miribel) sont les moins diversifiées, les premières disposant sans doute d'un espace vital trop restreint, les secondes soumises à des facteurs écologiques limitants. Des conditions apparemment plus favorables à l'espèce règnent dans une zone intermédiaire où est réalisé son optimum écologique, permettant le maintien de populations à grand effectif et donc d'un pool génique potentiellement diversifié.

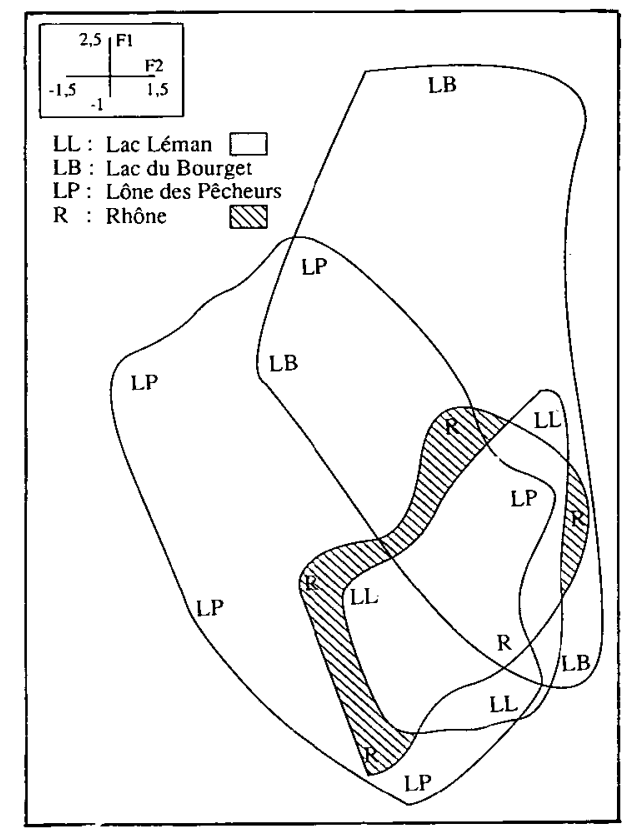

Figure 4 : Projection sur les plans F1-F2 des quatre nuages de points obtenus par analyse factorielle des correspondances multiples appliquée à l'ensemble des génotypes individuels de gardon.

Figure 4 : Projection on plane F1-F2 of the four groups of dots obtained by multivariate factorial analysis on all roach individual genotypes.

A part l'affinité entre petits cours d'eau, le dendrogramme des distances génétiques ne dégage pas de regroupements logiques. Il est certain qu'aucune conclusion définitive sur la stratégie de l'ombre commun ne pourra être tirée sans évaluation de l'impact des repeuplements. Une explication de la déstructuration par rapport à l'axe longitudinal pourrait se trouver dans les déversements de souches de pisciculture, puisque les introductions peuvent modifier les fréquences alléliques du pool génétique indigène (GUYOMARD et KRIEG, 1986). Cependant une analyse factorielle des correspondances multiples réalisée sur l'ensemble des échantillons récoltés à l'époque (SOEWARDI, 1988) a montré des génotypes de souches de pisciculture bien distincts de ceux des populations sauvages, ce qui conduit PERSAT (1988) à conclure que les souches introduites n'auraient guère eu de descendance. Sous réserve des résultats de l'étude complémentaire en cours sur ce point, il nous semble donc peu probable que les alevinages aient une influence sur la structure observée. Une autre cause possible de déstructuration està rechercher dans les aménagements du fleuve (endiguements, barrages, pollutions...) susceptibles de détruire des frayères, dégrader les conditions de croissance et ainsi diminuer le succès de la reproduction, conduisant à une réduction, voire une destruction, de populations. 


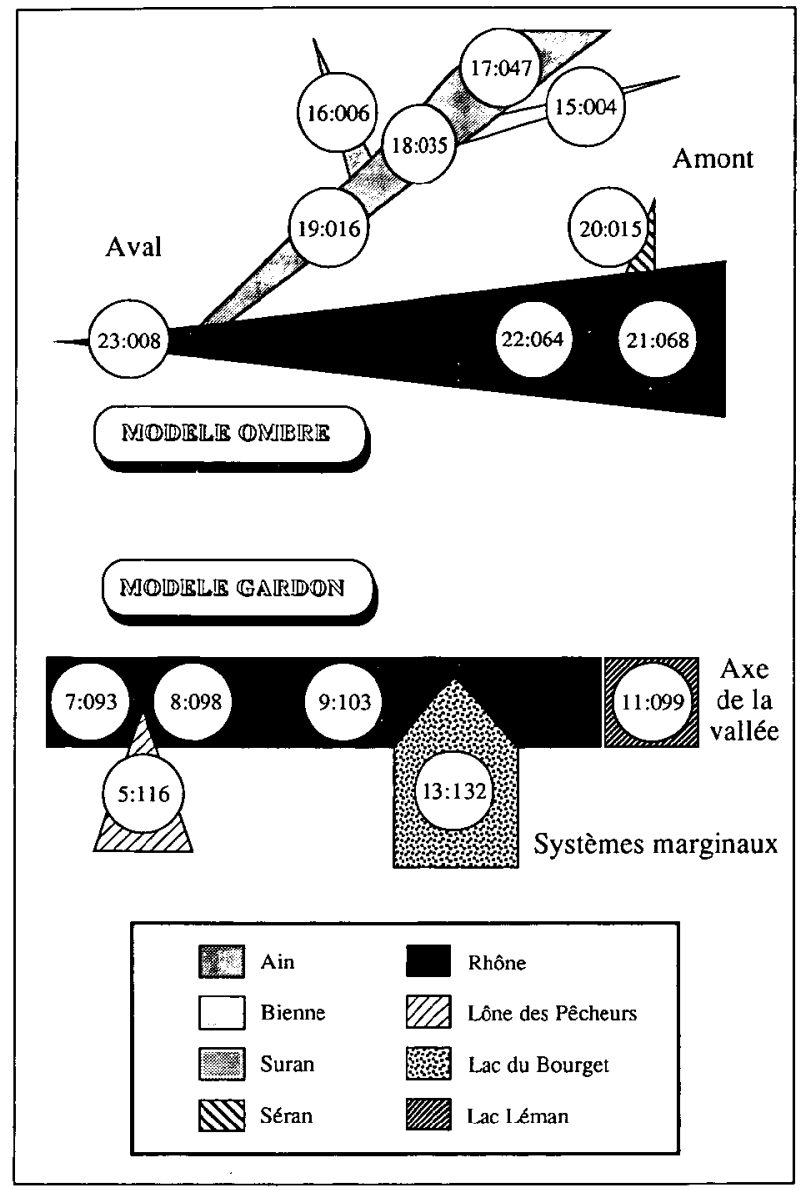

Figure 5 : Gradients d'hétérozygotie estimée (He) des deux espèces dans le réseau fluvial. La largeur des bandes représente cette diversité génétique. Dans les cercles, le premier nombre est le numéro de la station, le deuxième représente la valeur de He en millièmes d'unités.

Figure 5 : Heterozygosity $(\mathrm{He})$ gradients of both species within the fluvial network. The width of the lines represents this genetic diversity. In the circles, the first number refers to the station and the second is He ( 3 digits).

\section{CONCLUSION}

Nous avons pu constater dans cette étude un contraste dans l'organisation du pool génétique des espèces, mettant en évidence une exploitation spatiale différente du réseau fluvial. Le gardon, espèce limnophile, atteint son maximum de diversité dans les annexes du fleuve, écotones entre ce dernier et les marges de la vallée fluviale, à partir desquels il alimente le cours principal en génotypes sélectionnés dont la dérive vers l'aval maintient l'homogénéité longitudinale. Au contraire, s'accrochant davantage au terrain du chenal comme tout animal rhéophile, l'ombre tend à s'organiser en communautés autonomes (quelle qu'en soit l'origine naturelle ou artificielle) et que n'influence pas la dérive issue des populations de l'amont, témoin la grande distance génétique qui sépare les populations géographiquement proches de Chautagne et de Pont de Lucey.

Ces différences ont donc leur source dans les caractéristiques propres de chacune des deux espèces, dans l'adéquation entre leurs exigences écologiques et les caractéristiques des milieux qui les abritent, mais peut-être aussi dans la gestion des stocks pratiquée par les pêcheurs, essentiellement du fait des introductions. 


\section{REMERCIEMENTS}

Nous remercions les pêcheurs professionnels, et plus particulièrement $P$. BUISSON et M. SIMEON, pour leur aide dans la collecte des échantillons.

\section{BIBLIOGRAPHIE}

BOUVETY., SOEWARDI K. et PATTEEE., 1990. Genetic divergence within natural populations of grayling (Thymallus thymallus) from two French river systems. Archiv Hydrobiol., 119, 89-101.

BOUVET Y., SOEWARDI K. et PATTEE E., 1991. The discrimination of roach, Rutilus rutilus (Linnaeus, 1758) populations in different parts of a river system. An investigation using biochemical markers. Hydrobiologia, 209, 161-167.

GUYOMARD R. et KRIEG F., 1986. Mise en évidence d'un flux génique entre populations naturelles de truite fario et souche de repeuplement dans deux rivières de Corse. Bull. fr. Pêche Piscic., 303, 134-140.

KIMURA M., 1990. Théorie neutraliste de l'évolution. Flammarion, Paris, $472 \mathrm{p}$.

KRAIEM M. et PATTEE E., 1980. La tolérance à la température et au déficit en oxygène chez le Barbeau (Barbus barbus L.) et d'autres espèces provenant des zones piscicoles voisines. Arch. Hydrobiol., 88, 250-261.

NEI M., 1972. Genetic distance between populations. Am. Nat, 106, 283-292.

NEI M., 1973. Analysis of gene diversity in subdivided populations. Proc. natnI Acad. Sci. USA, $70,3321-3323$.

PATTEE E., 1988. Fish and their environment in large European river ecosystems. The Rhone. Sci Eau, 7, 35-74.

PASTEUR G., 1985. Les paramètres statistiques communément utilisés dans l'exploitation des résultats de l'électrophorèse des protéines et leur avenir en systématique. In Goyffon $\mathrm{M}$. et d'Hondt J.L. (eds), Electrophorèse et Taxonomie. Soc. zool. Fr., Mémoire 42, Paris, 141-180.

PASTEUR N., PASTEUR G., BONHOMME F., CATALAN J. et BRITTON-DAVIDIAN J., 1987. Manuel technique de génétique par électrophorèse des protéines. Tec \& Doc, Lavoisier, Paris, $217 \mathrm{p}$.

PERSAT H., 1976. Principaux aspects de l'écologie de l'ombre commun Thymallus thymallus (L. 1758) (Poissons Salmonidés). Thèse de Spécialité, Univ. Lyon-I, 69 p., bibl. \& annexes.

PERSAT H., 1988. De la biologie des populations de l'ombre commun Thymallus thymallus (L. 1758) à la dynamique des communautés dans un hydrosystème fluvial aménagé, le Haut-Rhône français. Eléments pour un changement d'échelles. Thèse d'Etat, Univ. Lyon-I, $223 \mathrm{p}$.

SNEATH P.H. et SOKAL R.R., 1973. Numerical taxonomy. Freeman, San Francisco CA, 573 p.

SOEWARDI K., 1988. Caractérisation des populations de Thymallus thymallus L. (Poissons Salmonidés) et de Rutilus rutilus L. (Poissons Cyprinidés) dans le réseau fluvial du Rhône. Approche par l'étude du polymorphisme enzymatique. Thèse Univ. Lyon-I, $125 \mathrm{p}$.

WRIGHT S., 1931. Evolution in Mendelian populations. Genetics, 16, 97-159. 